\title{
Erratum to: Weapon carrying and psychopathic-like features in a population-based sample of Finnish adolescents
}

\author{
Suvi Saukkonen ${ }^{1}$ Taina Laajasalo ${ }^{2,3} \cdot$ Markus Jokela $^{3}$ Janne Kivivuori ${ }^{4}$. \\ Venla Salmi ${ }^{4}$ Eeva T. Aronen ${ }^{1,2}$
}

Published online: 6 June 2015

(c) Springer-Verlag Berlin Heidelberg 2015

\section{Erratum to: Eur Child Adolesc Psychiatry DOI 10.1007/s00787-015-0724-2}

We regret that the Table 2 erroneously includes some negative signs that were not detected during copyediting. Please find the corrected Table 2 on the following page.

The online version of the original article can be found under doi:10.1007/s00787-015-0724-2.

Suvi Saukkonen

suvi.saukkonen@helsinki.fi

1 Clinical Institute, Clinic for Children and Adolescents, Child

Psychiatry, University of Helsinki, Helsinki University

Central Hospital, Tukholmankatu 8 C 613, 00290 Helsinki,

Finland

2 Child Psychiatry, Forensic Psychiatric Center for Children and Adolescents, Helsinki University Central Hospital, Helsinki, Finland

3 Institute of Behavioural Sciences, University of Helsinki, Helsinki, Finland

4 Criminological Unit, National Research Institute of Legal Policy, Helsinki, Finland 
Table 2 Descriptive statistics of the predictor variables of the study subgroups (knife, gun, other weapon, no weapon)

\begin{tabular}{|c|c|c|c|c|c|c|c|c|c|c|c|c|c|}
\hline & \multirow[t]{2}{*}{ Scale } & \multicolumn{3}{|c|}{$\begin{array}{l}\text { Knife } n=341(230 \\
\text { boys, } 110 \text { girls })\end{array}$} & \multicolumn{3}{|c|}{$\begin{array}{l}\text { Gun } n=25 \text { ( } 23 \text { boys, } \\
2 \text { girls })\end{array}$} & \multicolumn{3}{|c|}{$\begin{array}{l}\text { Other weapon } n=73 \\
\text { ( } 54 \text { boys, } 19 \text { girls) }\end{array}$} & \multicolumn{3}{|c|}{$\begin{array}{l}\text { No weapon } n=4416 \\
\text { ( } 2070 \text { boys, } 2346 \text { girls) }\end{array}$} \\
\hline & & Mean & SD & Range & Mean & $\mathrm{SD}$ & Range & Mean & $\mathrm{SD}$ & Range & Mean & $\mathrm{SD}$ & Range \\
\hline Delinquency, acts/year ${ }^{\mathbf{a}}$ & $0-7$ & 2.9 & 2.0 & $0-7$ & 4.2 & 2.7 & $0-7$ & 3.5 & 2.1 & $0-7$ & 0.8 & 1.2 & $0-7$ \\
\hline Victimization $^{\mathrm{a}}$ & $0-6$ & 1.7 & 1.5 & $0-6$ & 2.5 & 2.6 & $0-6$ & 1.5 & 1.6 & $0-6$ & 0.6 & 1.0 & $0-6$ \\
\hline Family functioning ${ }^{\mathrm{b}}$ & $11-55$ & 36.5 & 7.0 & $11-52$ & 32.1 & 8.7 & $11-44$ & 36.3 & 7.5 & $11-51$ & 41.2 & 5.9 & $14-55$ \\
\hline Perceived peer delinquency ${ }^{a}$ & $3-9$ & 7.4 & 1.9 & $3-9$ & 6.4 & 2.2 & $3-9$ & 7.3 & 2.0 & $3-9$ & 5.4 & 2.1 & $3-9$ \\
\hline Academic performance ${ }^{b}$ & $12-30$ & 22.1 & 3.3 & $12-30$ & 19.4 & 4.1 & $12-30$ & 21.4 & 3.2 & $15-30$ & 23.4 & 3.7 & $12-30$ \\
\hline Sense of security ${ }^{b}$ & $6-24$ & 20.1 & 3.1 & $6-24$ & 18.0 & 5.2 & $6-24$ & 19.6 & 4.4 & $6-24$ & 20.1 & 2.7 & $6-24$ \\
\hline APSD-SR total scores & $0-40$ & 16.1 & 5.5 & $3-36$ & 21.2 & 8.6 & $10-40$ & 17.3 & 5.3 & $8-30$ & 12.0 & 4.8 & $0-34$ \\
\hline
\end{tabular}

$A P S D$-SR antisocial process screening device-self report

${ }^{a}$ Higher score indicates worse situation

b Higher score indicates better situation 